\title{
Open-atmosphere structural depth profiling of multilayer samples of photovoltaic interest using laser-induced plasma spectrometry
}

\author{
S. Palanco , M. Gabás , L. Ayala , S. Bijani , E. Barrigóni, C. Algora , I. Rey-Stolle , J.R. Ramos-Barrado
}

\begin{abstract}
The present work aims to assess Laser-Induced Plasma Spectrometry (LIPS) as a tool for the characterization of photovoltaic materials. Despite being a well-established technique with applications to many scientific and industrial fields, so far LIPS is little known to the photovoltaic scientific community. The technique allows the rapid characterization of layered samples without sample preparation, in open atmosphere and in real time. In this paper, we assess LIPS ability for the determination of elements that are difficult to analyze by other broadly used techniques, or for producing analytical information from very low-concentration elements. The results of the LIPS characterization of two different samples are presented: 1) a 90 $\mathrm{nm}$, Al-doped ZnO layer deposited on a Si substrate by RF sputtering and 2) a Te-doped GaInP layer grown on GaAs by Metalorganic Vapor Phase Epitaxy. For both cases, the depth profile of the constituent and dopant elements is reported along with details of the experimental setup and the optimization of key parameters. It is remarkable that the longest time of analysis was $\sim 10 \mathrm{~s}$, what, in conjunction with the other characteristics mentioned, makes of LIPS an appealing technique for rapid screening or quality control whether at the lab or at the production line.
\end{abstract}

Index Terms - surface analysis, laser induced plasma spectrometry, in-situ.

\section{INTRODUCTION}

Traditional analytical techniques require the collection of samples, transportation and storage until the analysis is eventually done. Often, the chosen technique requires a procedure involving lengthy preparation steps before the actual measurement and data processing render the results available. Although it seems clear that the end user is provided with the best possible results in terms of accuracy and precision, the trade-off may have been fairly expensive in terms of response time. Moreover, such highly-accurate results might not be required. In many -if not most- cases the actual requirements fall among the following: fast-analysis times or real-time, in situ, non-destructive, non-invasive or low cost.

In other cases, the same well-established techniques are not capable to produce analytical results for a given element due to spectral interferences or to the lack of a source capable to excite the element as it is often the case for elements of the first period of the periodic table.

LIPS is a well established atomic emission spectrometry technique, with proven advantageous characteristics such as little or no need for sample preparation, fast analysis time and applicability to a wide variety of samples, whether conductive or insulating. The damage to the sample ranges from hundreds of microns to several millimeters in diameter which, in most cases, is negligible in comparison to the overall sample dimensions $[1,2]$.

In addition to these characteristics, the increasing availability of compact laser sources and miniaturized spectrometers make of LIPS a technique ideally suited for the development of in-plant characterization instruments. In fact, the growing demand for in-situ results has been accompanied by the increasing scientific efforts devoted to the research and development of field deployable analytical techniques and instruments. In this sense, atomic emission spectrometry has received a significant share of the work, the advances in spark emission spectrometry and laser-induced plasma spectrometry being the most remarkable $[3,4]$. Although both techniques rely on the same measurement principle, spark spectrometry is handicapped by the nature of the atom source it employs. Sparks of analytical quality are only attainable from clean metallic targets, a fact that significantly restrains the applicability range of the technique. In contrast, LIPS is well known for its wide applicability to all sort of samples, whether metallic or not, and additionally, the ablating action of the focused laser beam has been proved to be an excellent tool for cleaning and conditioning the surface prior to the analysis [5].

\section{A. LIPS fundamentals and method}

At the laser irradiances commonly used in LIPS (up to $10^{10}$ $\mathrm{W} \cdot \mathrm{cm}^{-2}$ ), laser interaction with matter starts through multiphoton $(n)$ ionization of an element, $M$ :

$$
M+n h v \rightarrow M^{+}+e^{-}
$$

which is the source for the first free electrons (if a small number not already present at the sample surface). Those initial electrons are then accelerated by the intense electric field of the laser pulse (inverse bremsstrahlung) fueling the production of more free electrons and ions through collisions in a cascade ionization process. On its expansion, the plasma front compresses the initially transparent surrounding gas whose temperature rises. As a result, it starts absorbing the laser radiation and from that point plays a critical role in the whole interaction phenomena. At an early stage, the energy is 
mostly transferred by conduction, while radiation becomes the dominant mechanism at a later stage.

It can be generally stated that, above an energy threshold, the ablated mass increases with the laser pulse energy to a point where the plasma becomes opaque and saturation in the rate of removal is reached. Parameters such as laser irradiance, focusing geometry, surface reflectivity, temperature, thermal conductivity and latent heats of fusion and vaporization govern the removal process both in terms of amount $\left(10^{-9}-10^{-5} \mathrm{~g} \cdot\right.$ pulse $^{-}$ $\left.{ }^{1}\right)$ and depth $\left(10^{-9}-10^{-4} \mathrm{~m} \cdot \mathrm{pulse}^{-1}\right)$.

The falling edge of the laser pulse signals the end of energy input and, hence, the beginning of plasma cooling through recombination, relaxation and emission of radiation. During the first stages, recombination and bremsstrahlung dominate the emission spectrum in the form of an intense continuum which is overlaid by broad emission from ionized species. As the plasma temperature and electron density decrease, so do the broadening and the intensity of emission lines. Between $0.5 \times 10^{-6}$ and $1 \times 10^{-6} \mathrm{~s}$ the continuum significantly lowers and line emission due to neutral species prevails.

The principle of analysis in LIPS is similar to those of other atomic emission spectrometries. The plasma emission in the UV-VIS is both spectrally- and time-resolved to identify the atomic (and sometimes molecular) species. A measurement implies the integration of the signal corresponding to a line or group of lines of the species of interest over a time period. The analysis result can be either semi-quantitative or quantitative. For the latter, the concentration of a sample constituent is determined by plotting a calibration curve of the signal versus the element's concentration in samples of known composition. Detection limits achievable are often in the ppm level.

Several advantages of surface characterization with laserinduced plasma spectrometry are the simplicity of the method, the possibility of simultaneously monitoring several elements in the plasma and the integration of the sampling and excitation stages into a single step. Additionally, the sample doesn't need to be transported to the instrument, but rather, the laser can be guided to form the plasma on the sample, a fact which is of particular interest for the purpose of this work.

\section{EXPERIMENTAL}

A Q-switched Nd:YAG (Quantel Brilliant B) laser operating on its fundamental wavelength $(1064 \mathrm{~nm})$, is guided and focused with a best-form plano-convex lens $(\mathrm{d}=25 \mathrm{~mm}, \mathrm{f}=$ $150 \mathrm{~mm}$ ) to the sample surface. The laser is capable of producing short pulses of up to $800 \mathrm{~mJ}$ at a fixed repetition rate of $10 \mathrm{~Hz}$ although the energy dose for each particular sample has been conditioned by extending the q-switch delay and spatial filtering of the beam.

The plasma is produced at open atmosphere and the light emitted is collected by means of two plano-convex spherical lenses $(\mathrm{d}=50 \mathrm{~mm}, \mathrm{f}=150 \mathrm{~mm}$ and $\mathrm{d}=50 \mathrm{~mm}, \mathrm{f}=200 \mathrm{~mm})$ and guided to a $163 \mathrm{~mm}$ focal length Czerny-Turner spectrograph
(Andor Shamrock 163i) fitted with a 25 micron entrance slit, a 1200 line $\mathrm{mm}^{-1}$ grating and a $2048 \times 512$ pixel intensified multichannel CCD detector (Andor iStar). This configuration allows an excellent performance, versatility and ease of alignment. Synchronization of excitation and detection is carried out with a digital delay and pulse generator (Stanford Research Systems DG535).

\section{SAMPLES}

Sample \#1. Al:ZnO films were deposited by RF sputtering of $\mathrm{ZnO}(99.99 \%)$ and $\mathrm{Al}_{2} \mathrm{O}_{3}(99.99 \%)$ ceramic targets. The temperature of the crystalline $\mathrm{Si}(100)$ substrate was $300^{\circ} \mathrm{C}$. The resulting sample has a $0.7 \%$ content of $\mathrm{Al}$ referred to $\mathrm{Zn}$. The $\mathrm{ZnO}$ layer is $90 \mathrm{~nm}$ in depth. Sample \#2. The semiconductor III-V layers were grown by MOVPE (Metal Organic Vapor Phase Epitaxy). The sample shown here consists of a $1000 \mathrm{~nm}$ GaInP:Te layer grown on a $1000 \mathrm{~nm}$ buffer GaAs layer on top of an undoped GaAs substrate.

\section{RESULTS AND DISCUSSION}

\section{A. Sample \#1}

In order to evaluate the applicability of the technique for spectral measurements in samples containing transparent layers, a sample (\#1) consisting of an $\mathrm{Al}$ doped $\mathrm{ZnO}$ layer on top of a $\mathrm{Si}$ substrate was chosen. The spectral window was centered at $300 \mathrm{~nm}$ seeking to simultaneously monitor the signals for the three elements of interest (Al, $\mathrm{Zn}$ and Si). Fig. 1 shows a LIP spectrum of sample \#1 where the atomic emission of $\mathrm{Al}, \mathrm{Zn}$ and $\mathrm{Si}$ is labeled. It is important to remark the excellent Signal-to-Noise Ratio (SNR) of the spectrum and that it was acquired from a single laser shot.

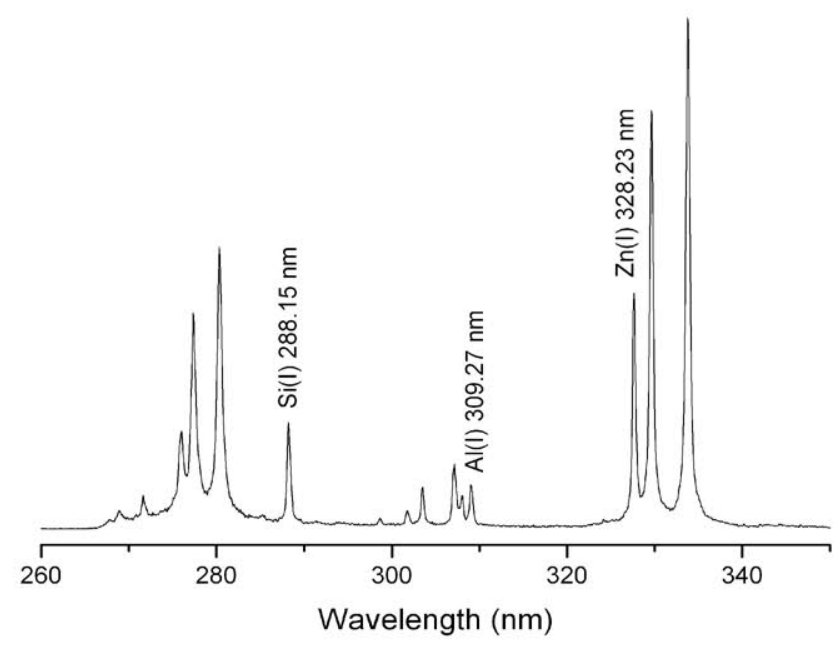

Fig. 1. LIP spectrum of sample \#1 ( $\mathrm{ZnO}: \mathrm{Al}$ on a $\mathrm{Si}$ substrate) showing the emission of the main constituents. Acquisition delay $500 \mathrm{~ns}$, gate $1000 \mathrm{~ns}$. Time of analysis $0.1 \mathrm{~s}$. 


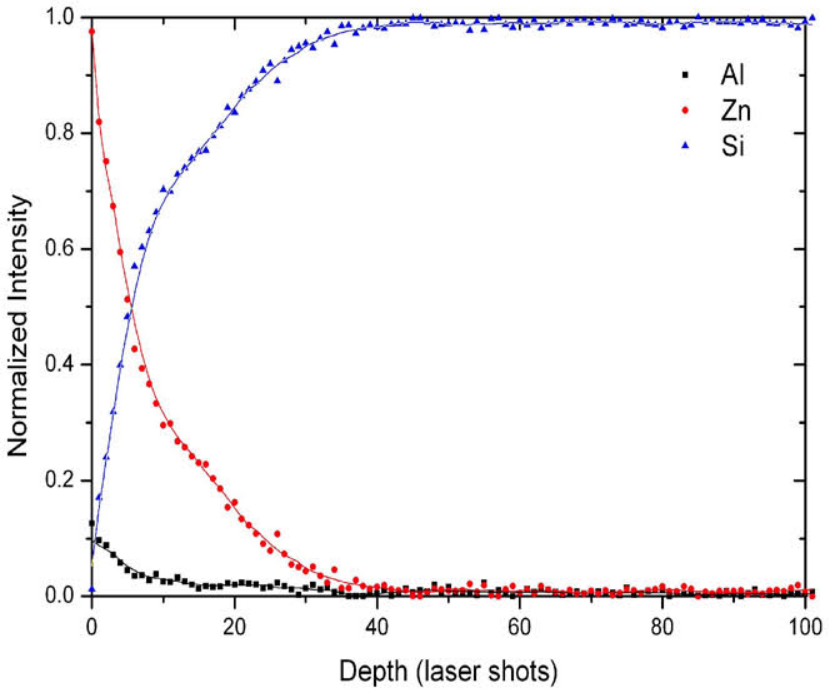

Fig. 2. Depth profile of Sample \#1 obtained under the same experimental conditions as the spectrum in Fig. 1. The top $\mathrm{ZnO}: \mathrm{Al}$ had a $90 \mathrm{~nm}$ thickness. Al is plotted at $10 \mathrm{x}$ intensity to improve visibility by the reader. Time of analysis $10 \mathrm{~s}$.

Given that the laser removes -or ablates- a fine layer of material, by repeating the above process on the same sample spot a compositional depth profile can be obtained. In order to achieve a better in-depth resolution, the laser pulse energy is lowered in order to reduce the average ablation rate (AAR). By doing so, the convolution of information from different depths in the sample is also minimized. For the following measurements, the laser q-switch delay was elongated to achieve a fluence of $9.0 \mathrm{~J} \cdot \mathrm{cm}^{-2}$ at the sample.

Fig. 2 shows the depth profile obtained for sample \#1. As shown, 100 pulses were fired on the same position of the sample. Each point represents a separate laser shot. Given the good precision of the data shown, it would have been possible to further reduce the laser energy and, as a consequence, to improve the in-depth resolution of the technique without requiring statistical processing of the data. Average ablation rates in the order of several nanometers per laser shot are not uncommon to the technique [6].

A closer view at Fig. 2 reveals some mixing between the two layers, in particular the tails of $\mathrm{Zn}$ and $\mathrm{Al}$ are longer that what the Gaussian error function -which describes the profile of an abrupt interface- would predict. There are two factors contributing to this behavior, namely the laser beam shape and the contribution from the crater walls. A behavior closer to the ideal error function is obtained when the laser beam used has a flat cross-sectional energy profile. In our case, the beam energy profile was Gaussian and, although only a central portion was used, the Gaussian shape is partially recreated at the surface.

In the other hand, after a certain number of laser pulses, the crater becomes deep and its walls may pose a constrain to plasma expansion. For craters with a high depth-to-width aspect ratio the plasma obtained is hotter and optically thicker.
This effect, known as plasma shielding will result in the AAR decreasing as the crater becomes deeper. This is usually circumvented by focusing the beam to a larger spot in the sample. The advantage of this practice is double as it also improves the surface sensitivity by sampling a wider area. Nevertheless, interaction between the plasma and the crater walls still occurs, and a small amount of wall material is removed resulting in depth profiles which exhibit longer tails.

\section{B. Quantitative vs. semi-quantitative}

It is also important to note that, as with other atomic emission techniques, a calibration procedure has to be carried out in order to produce quantitative results. Calibration of concentration involves feeding the instrument with a set of samples whose composition is similar to the samples of interest. For complex samples, the calibration function of a given element depends not only on the concentration of said element but also on the concentration of the remaining sample constituents. As a result, calibration curves are best obtained using multivariate calibration on a sample set diverse enough to cover the concentration space of interest $[3,4]$.

However, in many cases, a mere semi-quantitative result like those presented here is sufficient to extract a meaningful idea of the sample composition and the presence of dopants or contaminants. Moreover, it must be highlighted that, whether quantitative or not, such results were obtained without sample preparation and in a $10 \mathrm{~s}$ period only. The time of analysis per shot is actually even shorter than what this figure suggests as the signal integration time -or gate- was 1000 ns. Time-wise, the bottlenecks usually found in LIPS are due to the laser repetition rate and the maximum acquisition rate of the detector -in frames per second. All in all, spectra of analytical quality can be acquired up to kilohertz rates by making use of the appropriate hardware [7].

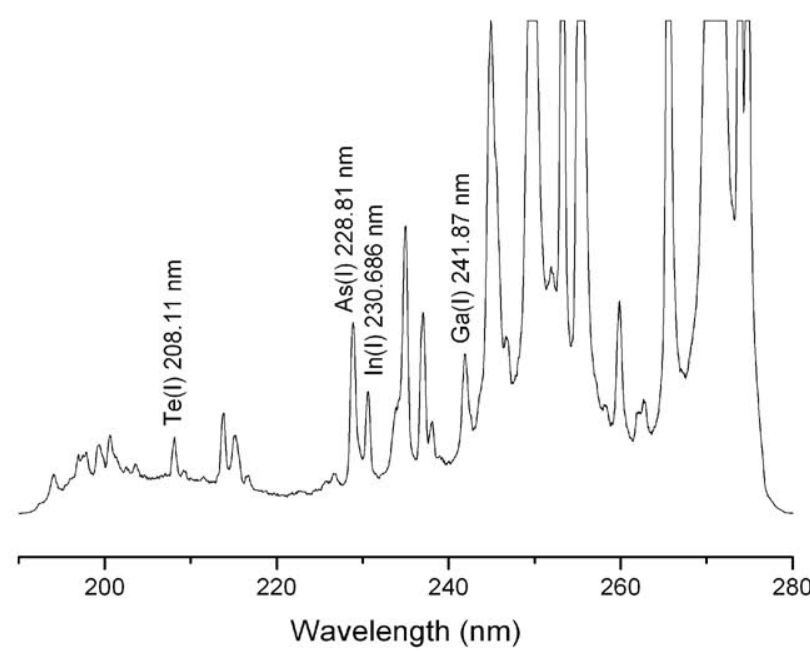

Fig. 3. LIP spectrum of sample \#2 (GaInP:Te on a GaAs buffer layer on a GaAs substrate) showing the emission of the main constituents. Acquisition delay 500ns, gate $1000 \mathrm{~ns}$. The portion of the spectrum beyond $245 \mathrm{~nm}$ is deliberately saturated. Time of analysis $0.1 \mathrm{~s}$. 


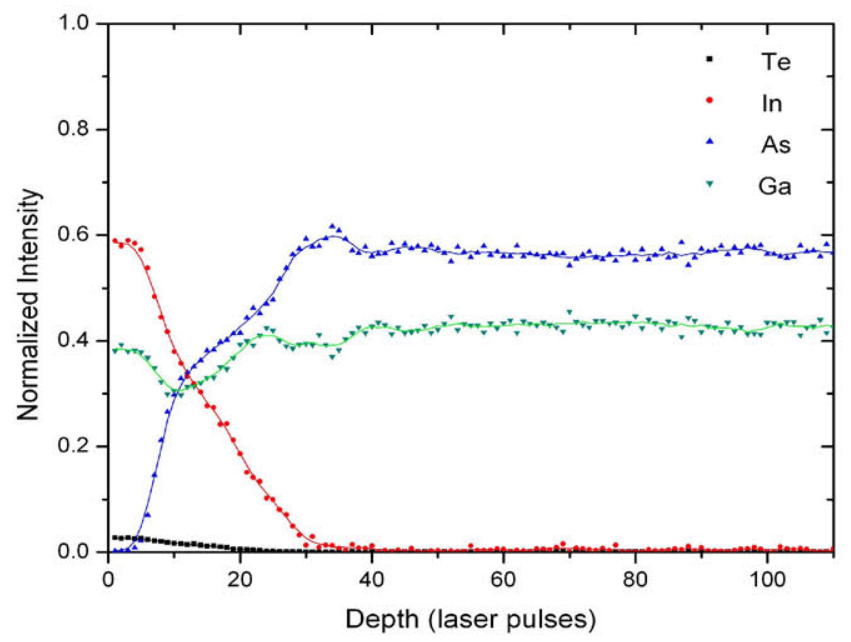

Fig. 4. Depth profile of sample \#2 obtained under the same experimental conditions as the spectrum in Fig. 3. The thickness of the top GaInP:Te was $1000 \mathrm{~nm}$ with a further $1000 \mathrm{~nm} \mathrm{GaAs}$ buffer layer on top of the GaAs substrate. Time of analysis $11 \mathrm{~s}$.

\section{Sample \#2}

In this section, a further example of the potential of LIPS is illustrated for the detection of doping elements like Te. Sample \#2 has a topmost GaInP:Te layer on top of a GaAs matrix. Fig. 3 shows a single-shot laser-induced spectrum of Sample \#2. In this case, the spectral window was centered at $237 \mathrm{~nm}$ in order that emission lines for Te, Ga, In and As could be simultaneously registered. Although $\mathrm{P}$ emission lines are present, they are severely interfered by other lines in the spectrum. Using a wider spectral window would overcome this issue but, in our case, it was fixed by the instrument available. In any case, the spectrum shows the emission for the remaining constituents of the sample, the presence of the Te (I) emission at $208.11 \mathrm{~nm}$ with good SNR being remarkable as the concentration of this element in the sample is $9.0 \times 10^{18} \mathrm{~cm}^{-3}$.

Fig. 4 is a depth profile of Sample \#2. The plots for the different elements are consistent with the composition of the sample and further illustrate the potential of LIPS for a rapid monitoring of sample composition with neither sample preparation nor vacuum requirements. As with the results shown in Fig. 2, this semi-quantitative profile can be turned into a fully quantitative compositional profile by building a calibration curve for each element as well as for the depth axis (z), shown in number of pulses both in Fig. 2 and Fig. 4.

\section{Depth calibration}

Although it is out of the scope of this work, calibration of the depth axis can be carried out using a set of layered samples whose layer depths and composition are known. The number of laser pulses necessary to reach the different interfaces is monitored and used to fit a function of depth versus number of pulses [8]. This procedure is not straightforward -it implies that the AAR is known throughout the sample depth. In turn, as the reader can infer from the Quantification and Introduction sections, AAR is greatly dependent on sample composition. Elements with low melting and vaporization heats -like $\mathrm{Zn}$ or Te- exhibit much higher AAR than those which are more energy demanding like $\mathrm{Si}$, or $\mathrm{Al}$. This is of particular importance when the laser reaches an interface between two layers since a transition will occur from the AAR of the top layer to that of the bottom layer.

\section{CONCLUSIONS}

The capability to produce instant analytical results without sample preparation and without vacuum requirements makes of LIPS an appealing technique for PV applications, not only in the lab but also at the production environment. Simultaneous determination of dopant and matrix elements which are difficult to analyze by other broadly used techniques, is possible in open atmosphere. Semi-quantitative depth profiles of two samples of interest to the PV industry have been produced with times of measurement lower than 12 $\mathrm{s}$ in all cases. Work is already underway to upgrade our laser and produce cleaner profiles. In a further work, the semiquantitative profiles will be turned into fully quantitative compositional profiles.

\section{ACKNOWLEGMENTS}

This work has been supported by the Spanish Ministerio de Economía y Competitividad with the projects TEC201128639-C02-01 and -02 . and with the INNPACTO program by means of the IPT-2011-1441-920000 and IPT-2011-1408420000 projects. This research has been also supported by the Comunidad de Madrid under the NUMANCIA II program (S2009/ENE1477) and FEDER funds. of the EC. S.P. thanks the Spanish Ministerio de Ciencia e Innovación for research contract RYC-2010-06711 under the Ramón y Cajal program.

\section{REFERENCES}

[1] D.A. Rusak, B.C. Castle, B.W. Smith, J.D. Winefordner, "Fundamentals and applications of laser-induced breakdown spectrometry", Crit. Rev. Anal. Chem. Vol 27, pp 257-266, 1997.

[2] D.A. Cremers, A.K. Knight, "Laser induced breakdown spectrometry", in "Encyclopedia of Analytical Chemistry", R.A. Meyers (Ed.), Wiley, Chichester, 2000.

[3] S. Palanco, J.J. Laserna, "Full automation of a laserinduced breakdown spectrometer for quality assessment in the steel industry with sample handling, surface preparation and quantitative analysis capabilities", J. Anal. At. Spectrom., Vol 15, pp 1321-1327, 2000.

[4] S. Palanco, A. Alises, J. Cuñat, J. Baena, J.J. Laserna, "Development of a portable laser-induced plasma spectrometer with fully-automated operation and quantitative 
analysis capabilities", J. Anal. At. Spectrom., Vol 18, pp 933938, 2003.

[5] D.A. Cremers, L.J. Radziemsky, "Handbook of laserinduced breakdown spectroscopy" John Wiley \& Sons Ltd. Chichester, UK, 2006.

[6] J.M. Vadillo, C.C. García, S. Palanco, J. Laserna, "Nanometric range depth-resolved analysis of coated steels using laser- induced breakdown spectrometry with a $308 \mathrm{~nm}$ collimated beam", J. Anal. Atom. Spectrom. Vol 13, pp 793797, 1998.

[7] C. Lopez-Moreno, K. Amponsah-Manager, B.W. Smith, I.B. Gornushkin, N. Omenetto, S. Palanco, J.J. Laserna, J.D. Winefordner, "Quantitative analysis of low-alloy steel by microchip laser induced breakdown spectroscopy", J. Anal. At. Spectrom., Vol 20, pp 552-556, 2005.

[8] J. Cuñat, S. Palanco, F. Carrasco, M.D. Simón, J.J. Laserna, "Portable instrument and analytical method using laser-induced breakdown spectrometry for in situ characterization of speleothems in karstic caves", J. Anal. At. Spectrom., Vol 20, pp 295-300, 2005. 